\title{
microRNA-132 inhibits the proliferation, migration, and invasion of ovarian cancer cells by regulating CT10 oncogenic gene homolog II-related signaling pathways
}

\author{
Haiyan Jiang ${ }^{1,2 \#}$, Min Dai ${ }^{1 \#}$, Yao $\mathrm{Wu}^{2}$, Yansong Dong ${ }^{2}$, Lei $\mathrm{Qi}^{2}$, Qinghua $\mathrm{Xi}^{1}$, Guiwen Liang ${ }^{2}$ \\ ${ }^{1}$ Department of Obstetrics and Gynecology, ${ }^{2}$ Department of Emergency Medicine, Affiliated Hospital of Nantong University, Nantong, China \\ Contributions: (I) Conception and design: H Jiang, M Dai; (II) Administrative support: Q Xi; (III) Provision of study materials or patients: G Liang; \\ (IV) Collection and assembly of data: Y Wu, Y Dong; (V) Data analysis and interpretation: L Qi; (VI) Manuscript writing: All authors; (VII) Final \\ approval of manuscript: All authors. \\ \#These authors contributed equally to this work. \\ Correspondence to: Qinghua Xi and Guiwen Liang. 20 West Temple Road, Department of Obstetrics and Gynecology, Affiliated Hospital of Nantong \\ University, Nantong 226001, China. Email: ntdxfsyyxqh@163.com; liang_gui_wen@126.com.
}

\begin{abstract}
Background: Despite a large amount of evidence showing the involvement of microRNA-132 (miR-132) in the occurrence and prognosis of many different types of cancer, the role of miR-132 in ovarian cancer and its potential molecular mechanism have yet to be fully explained.

Method: We studied the biological function and molecular mechanism of miR-132 in ovarian cancer cell lines and clinical tissue samples using quantitative reverse transcription polymerase chain reaction (qRTPCR), Western blot, Luciferase reporter assay, CCK8 test, colony formation test, and scratch and Transwell assays.

Results: The expression level of miR-132 was significantly reduced in ovarian cancer cell lines and clinical tissue samples. When the level of miR-132 was increased, the proliferation, colony-forming, migration, and invasion abilities of ovarian cancer cells were significantly inhibited. We found that miR-132 inhibits the expression of transcription factor CT10 Oncogenic Gene Homologue II (CRKII) through specific targeting of mRNA 3'-UTR. We also observed a significant increase in CRKII expression in ovarian cancer. Notably, CRKII expression was negatively correlated with miR-132 expression in clinical ovarian cancer tissue. Down-regulation of CRKII had a similar inhibitory effect on miR-132 overexpression in ovarian cancer cells, while excessive expression of CRKII reversed the inhibitory effect mediated by the excessive expression of miR-132.
\end{abstract}

Conclusions: miR-132 inhibits the proliferation, invasion, and migration abilities of ovarian cancer cells through targeting CRKII.

Keywords: microRNA-132 (miR-132); ovarian cancer; CT10 Oncogenic Gene Homologue II (CRKII)

Submitted May 21, 2020. Accepted for publication Jul 08, 2020.

doi: $10.21037 /$ tcr-20-2435

View this article at: http://dx.doi.org/10.21037/tcr-20-2435

\section{Introduction}

Ovarian cancer has the highest incidence of any malignancy of the female reproductive system and also ranks first for mortality out of all gynecological tumors $(1,2)$. Accounting for $4.3 \%$ of female cancer deaths each year, the disease poses a serious threat to women's lives. At present, surgical resection plus adjuvant chemotherapy is still the preferred treatment method for ovarian cancer. However, the 5-year survival rate and high rates of recurrence and metastasis still present problems for patients after treatment (3). Therefore, there is an urgent need for new diagnostic and therapeutic targets for ovarian cancer to be identified. 
MicroRNA is a small non-coding single-stranded RNA measuring around 19-25 nt (nucleotides) in length, which is ubiquitous in animals and plants $(4,5)$. It binds specifically to the target RNA sequence, causing degradation or translation inhibition of target RNA, thus regulating gene expression and participating in a series of biological processes (6). microRNA-132 (miR-132) is a current hotspot of microRNA research. miR-132 is down-regulated in lung cancer, gastric cancer, ovarian cancer, and other malignant tumors, and it is also closely related to cancer occurrence and development (7). Currently, there are few studies on the specific regulatory mechanisms involved in the occurrence and development of ovarian cancer. Therefore, this paper will explore the role of miR-132 in regulating proliferation, metastasis, and invasion in ovarian cancer. miR-132 is expected to become a new target for the inhibition of malignant tumors.

CT10 Oncogenic Gene Homolog II (CRKII) is a key member of the CRK family, which is an important class of intracellular signal junction proteins that includes v-CRK, CRKI, CRKII, and CRKL (CRK-like). Structurally, CRK family members contain $\mathrm{SH} 2$ and $\mathrm{SH} 3$ domains, which mediate protein-protein interaction (8) and connect upstream and downstream signal transducers. In recent years, an increasing number of studies have shown that the expression of CRKII is closely related to tumor occurrence and development $(9,10)$. Recent research has confirmed the significant upregulation of CRKII in ovarian cancer cell line SKOV3, with its overexpression promoting cell proliferation. Meanwhile, knock down of CRKII with siRNA technology yielded the opposite result. Thus, this study aimed to investigate miR-132's role in ovarian cancer and its underlying mechanisms.

\section{Methods}

\section{Patients and tissue samples}

Forty tissue specimens from patients who were diagnosed with primary ovarian cancer between June 2010, to June 2019, were selected from the Department of Obstetrics and Gynecology, Affiliated Hospital of Nantong University. None of the patients had received any form of cancerspecific therapy before surgery. Normal ovarian specimens were taken from preventive hysterectomy and accessories. All specimens were obtained with the consent of the patient. Each tissue sample was frozen in liquid nitrogen immediately and stored at $-80^{\circ} \mathrm{C}$ before RNA extraction. This study was conducted in adherence with the guidelines of the Declaration of Helsinki (as revised in 2013) and received approval from the Ethics Committee of Nantong University (2019-L060). Written informed consent was obtained from all patients.

\section{Cell culture}

We obtained human ovarian surface epithelial cell line (HOSEpiC) and human ovarian cancer cell lines (SKOV3, OVCAR3, and HO-8910) from the Institute of Model Culture, Chinese Academy of Sciences (Shanghai). We cultured the cells in Roswell Park Memorial Institute (RPMI) 1640 (Gibco, Grand Island, NY, USA) with 10\% fetal bovine serum (FBS; Jake Bio, Shanghai, China), penicillin, and streptomycin at $37^{\circ} \mathrm{C}$ in an atmosphere of $5 \%$ $\mathrm{CO}_{2}$ and $90 \%$ humidity.

\section{Cell transfection}

miR-132 mimics and negative control (NC), siRNA against CRKII (si-CRKII), and siRNA against negative control (siNC) plasmid were purchased from Gene Pharmaceutical Company (Shanghai, China). Polymerase chain reaction (PCR) was performed to amplify the gene encoding CRKII from human genomic DNA before it was cloned into pcDNA3 vector (pcDNA3-CRKII). According to the manufacturer's protocol, Lipofectamine ${ }^{\circledR} 2000^{\mathrm{TM}}$ (final concentration, $50 \mathrm{nM}$ ) was used to transfect SKOV3 and HO-8910 cells with miR-132 mimics, NC, si-CRKII, and si-NC. After culturing the transfected cells in complete medium for 48 hours, the transfection efficiency was measured.

\section{Quantitative reverse transcription polymerase chain reaction (qRT-PCR)}

Total RNA was extracted from cells and tissues with Trizol reagent. Then, cDNA was synthesized for 30 minutes at $37^{\circ} \mathrm{C}$ with an $\mathrm{SYBR}^{\circledR}$ PrimeScript $^{\mathrm{TM}} \mathrm{RT}$ PCR kit (Takara Biotechnology, Ltd., Shanghai) following the protocol of the manufacturer. miR-132 expression was quantitated with a TaqMan miRNA assay kit (Applied Biosystems, Forster City, CA, USA) and miR-132 and U6 primers (Applied Biosystems) using an ABI 7900 system (Applied Biosystems). To quantitatively analyze CRKII, cDNA was synthesized using the PrimeScript RT reagent Kit (Takara, Dalian, China) following the instructions of the manufacturer. PCR was carried out with the ABI 7900 rapid system using SYBR Premix Ex 
Taq (TaKaRa). The following primers were used: miR132, forward primer: 5'-ACACTCCAGCTGGGTAACA GTCTACAGCCA-3', reverse primer: 5'-GTGTCGTG GAGTCGGCAATTCAGTTGAG-3'. CRKII forward, 5'-GCGGAGTAGCTGGTACTGG-3' andreverse, 5' GCGCGAGTTCTCTGAGACG-3'. The $2^{-\Delta \Delta C t}$ method was used to determine miR-132 and CRKII expression levels, with $\mathrm{U} 6$ and $\beta$-actin as internal standards.

\section{Western blot}

Total protein was extracted on ice for 30 minutes with RIPA cleavage buffer containing protease inhibitor (1\% NP40, 0.1\% sodium dodecyl sulfate (SDS), $100 \mu \mathrm{g} / \mathrm{mL}$ benzenesulfonyl fluoride, $0.5 \%$ sodium deoxycholate (PBS). The supernatant was then centrifuged at 13,000 $\times \mathrm{g}$ at $4{ }^{\circ} \mathrm{C}$ for 20 minutes. The BCA method was used to determine the protein concentration, and $10 \%$ sodium dodecyl sulfate-polyacrylamide gel (10\%SDS-PAGE) was used to separate $20 \mu \mathrm{g}$ of total protein. The proteins were then transferred to polyvinylidene difluoride membranes (PVDF, GEFAN BIOTECHNOLOGY, Shanghai, China). The membrane was sealed with TBST solution containing $20 \mathrm{mM}$ Tris-HCl, $\mathrm{pH} 7.5,150 \mathrm{mM} \mathrm{NaCl}$ and $0.1 \%$ Tween-20 with $5 \%$ skim milk for 1 hour at room temperature, before incubation overnight at $4{ }^{\circ} \mathrm{C}$ with primary antibodies against CRKII (1:500, Santa Cruz, CA, USA) or against $\beta$-actin (1:5,000, Santa Cruz). Finally, the membrane was washed, incubated with goat anti-mouse secondary antibody (mouse. no. Ab97040; 1:4,000; Abcam) bound to horseradish peroxidase (HRP), and placed at room temperature for 2 hours, with $\beta$-actin used as loading control. Protein bands were detected using enhanced chemiluminescence (ECL) luminol reagent (PerkinElmer Inc., USA).

\section{Cell proliferation and colony formation}

Cell proliferation was analyzed by culturing transfected cells for 48 hours. Cells in good growth condition were prepared into a cell suspension of 20,000 cells $/ \mathrm{mL}$. Then, $100 \mu \mathrm{L}$ of cells was added into a 96-well cell culture plate, with $2 \times 10^{3} / 100 \mu \mathrm{L}$ cells added into each well. After 24 hours of pre-culture in an incubator at $37^{\circ} \mathrm{C}, 10 \mu \mathrm{L}$ of Cell Counting Kit-8 CCK-8 (GEFAN BIOTECHNOLOGY, Shanghai, China) solution was added to the wells. Then, the cells were incubated at $37^{\circ} \mathrm{C}$ for 24,48 , or 72 hours. The absorbance was measured at $450 \mathrm{~nm}$ wavelength. In the colony-forming experiment, transfected cells were inoculated into 6-well plates with 500 cells per well before culture for 10 days. After fixing with methanol and staining with $1 \%$ crystal violet (Sigma), the colonies were counted with an optical microscope (Leica, Germany).

\section{Migration and invasion assays}

A scratch assay was performed to investigate cell migration. Approximately $5 \times 10^{5}$ cells were inoculated into 6 orifice plates. The cells were cultured in an incubator at $37^{\circ} \mathrm{C}$ with $5 \% \mathrm{CO}_{2}$ until they began to adhere to the wall of the culture dish. Scratches were created using the tip of a sterile $10 \mu \mathrm{L}$ pipette. PBS was used to wash the scratched cells off, and they were added to a serum-free medium. Samples and photographs were taken at 0,12 , and 24 hours. Finally, the migration distances of the cells were calculated.

For the Transwell assay, we used 24 borehole inserts with apertures of 8 microns (BD Biosciences, New Jersey, USA). Approximately $5 \times 10^{5}$ cells/holes were resuspended in $200 \mu \mathrm{L}$ medium without FBS and inoculated into the upper chamber coated with matrix gel. In addition, we added a complete culture medium of $500 \mu \mathrm{L}$ RPMI-1640 with $10 \%$ fetal bovine serum to the substrate and incubated at $37{ }^{\circ} \mathrm{C}$ and $5 \% \mathrm{CO}_{2}$. After 48 hours, dye with $0.1 \%$ crystal violet at room temperature for 10 minutes. After gently removing the upper layer cells with a cotton swab, we calculated the number of cells on each layer using an inverted microscope. Each test was performed in triplicate.

\section{Luciferase reporter assay}

We amplified 3'-UTR of wild-type CRKII by RT-PCR, including the binding site with miR-132, and cloned it into the downstream reporter plasmid pGL3 (Promega, Madison, WI, USA) of the luciferase reporter gene. QuickChange Site-Directed Mutage-nesis kit (Stratagene, La Jolla, CA, USA) was used to construct the 3'-UTR of mutant-type CRKII. A luciferase assay was performed by placing HO-8910 cells into 96 -well plates with $5 \times 10^{3}$ cells per well. Following 24 hours of culture, the cells were transfected with either $50 \mathrm{nM}$ miR-132 mimic or miR-NC mimic and $500 \mathrm{ng}$ wild-type CRKII 3'-UTR or mutant-type CRKII 3'-UTR plasmid, according to the manufacturer's instructions. After 48 hours of transfection, luciferase activity was measured using a dual luciferase reporter system following the protocol of the manufacturer. Renilla luciferase was employed for normalization. 


\section{A}
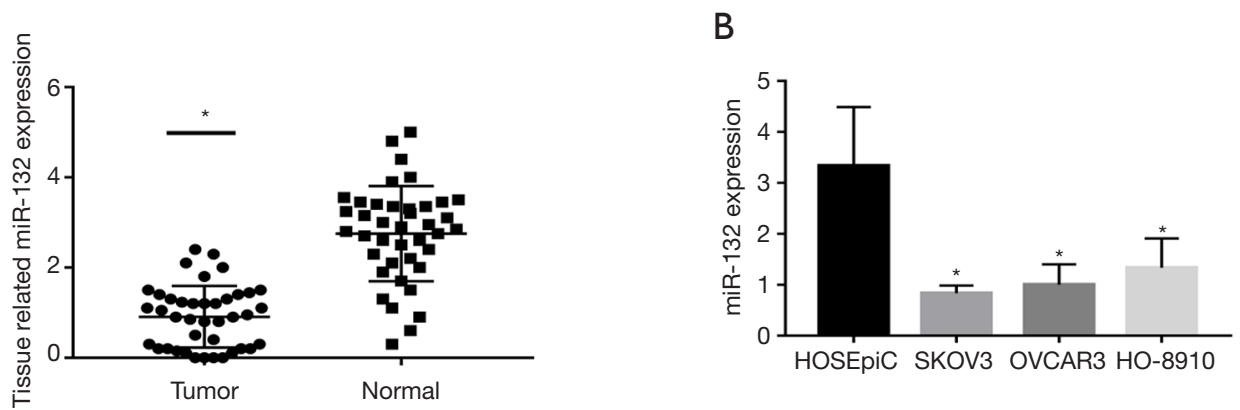

Figure 1 miR-132 expression was down-regulated in ovarian cancer cells and tissues. (A) qRT-PCR was used to detect tissue related miR132 levels in 40 cases of ovarian cancer and corresponding normal tissues. * $\mathrm{P}<0.05$ compared with normal samples. (B) miR-132 expression in human ovarian epithelial cells (HOSEpiC cells) compared with three human ovarian cell lines (OVCAR3, SKOV3, and HO-8910). *, $\mathrm{P}<0.05$ compared with HOSEpiC. miR-132, microRNA-132; qRT-PCR, quantitative reverse transcription polymerase chain reaction; HOSEpiC, human ovarian surface epithelial cell line.

\section{Results}

The expression of miR-132 was down-regulated in ovarian cancer cells and tissues

The expression of miR-132 was detected in 40 ovarian cancer tissues and normal ovarian tissue by qRT-PCR. There was a significantly lower expression of miR-132 in the cancerous tissue samples than in the normal samples $(\mathrm{P}<0.05)$ (Figure 1A). Then, miR-132 expression was detected in ovarian cancer and normal ovarian surface cell lines. In comparison with normal ovarian epithelial cell lines, the ovarian cancer cell lines had significantly down-regulated miR-132 expression (Figure 1B). The HO-8910 ovarian cancer cell line was selected for further study.

\section{miR-132 inbibited the growth of ovarian cancer cells}

To investigate the effect of miR-132 on cell proliferation and colony formation. we transfected HO-8910 cells with miR-132 mimics and miR-NC. CCK8 and colony formation assays were used to determine cell proliferation and colony formation. The level of miR-132 in the HO8910 cells transfected with miR-132 mimic was nearly four times higher than that in the miR-NC-transfected cells $(\mathrm{P}<0.05$, Figure $2 A)$. The CCK8 assay demonstrated that miR-132 overexpression significantly suppressed cell proliferation $(\mathrm{P}<0.05$, Figure $2 B)$. Colony formation analysis showed that when overexpressed, miR-132 limited the number of colonies in comparison with miR-NC $(\mathrm{P}<0.05$, Figure 2C).

\section{miR-132 inbibits the migratory and invasion ability of ovarian cancer cells}

The effects of miR-132 on the migration and invasion of human ovarian cancer cells were determined by scratch and perforation assays. Wound healing was significantly inhibited in HO-8910 cells with miR-132 overexpression compared with those treated with miR-NC (Figure 3A,B). The results of the Transwell assay showed that the invasion rate of HO-8910 cells was significantly lower than that of NC cells due to the overexpression of miR-132.

\section{miR-132 directly targets CRKII in ovarian cancer}

To further study the mechanism underlying the development of ovarian cancer, we found that the seed sequence of miR-132 was complementary to that of the 3'UTR of CRKII at 535-541 (Figure 4A). To verify whether CRKII is the direct target of miR-132, a luciferase assay was carried out. The activity of wild-type CRKII-3'UTR reporter was significantly decreased in H0-8910 cells transfected with miR-132 mimic compared to those transfected with miR-NC $(\mathrm{P}<0.05$, Figure $4 B)$. Meanwhile, miR-132 mimic did not inhibit the activity of mutant-type CRKII-3'-UTR reporter (Figure 4B). This indicates that CRKII might be the target of miR-132.

Then, to further clarify the relationship between miR-132 and CRKII, qRT-PCR and Western blot were performed to verify the mRNA and protein expression of CRKII. The results revealed that the CRKII expression at the levels of mRNA $(\mathrm{P}<0.05$, Figure $4 C)$ and protein (Figure $4 D$ ) was significantly reduced by miR-132 

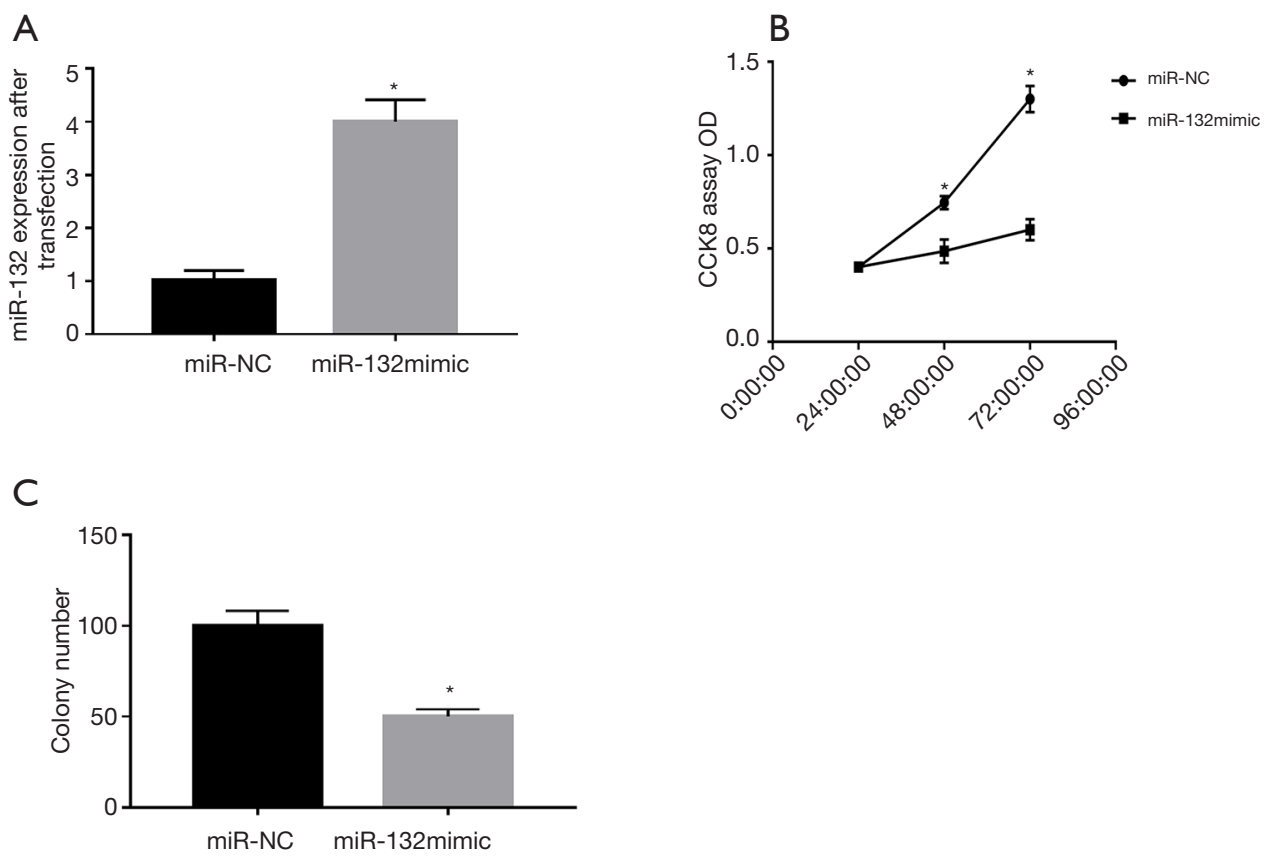

Figure 2 miR-132 inhibits the proliferation and colony formation of ovarian cancer cells. (A) The inhibitory effect of miR-132 on miR-NC expression in H0-8910 cells was detected with qRT-PCR. *, $\mathrm{P}<0.05$ compared with miR-NC. (B) The proliferation of H0-8910 cells was detected by CCK8 between 24 and $72 \mathrm{~h}$ after transfection with miR-NC or miR-132 mimic. *, $\mathrm{P}<0.05$, in comparison with miR-NC. (C) The results of the colony formation assay with HO-8910 cells transfected with miR-132 or miR-NC. *, P<0.05 compared with miR-NC. miR-132, microRNA-132; qRT-PCR, quantitative reverse transcription polymerase chain reaction.

A

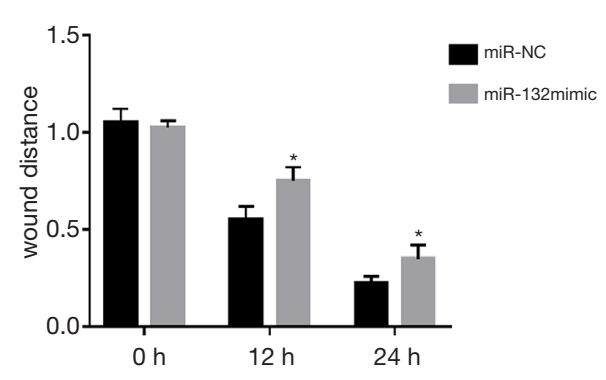

B

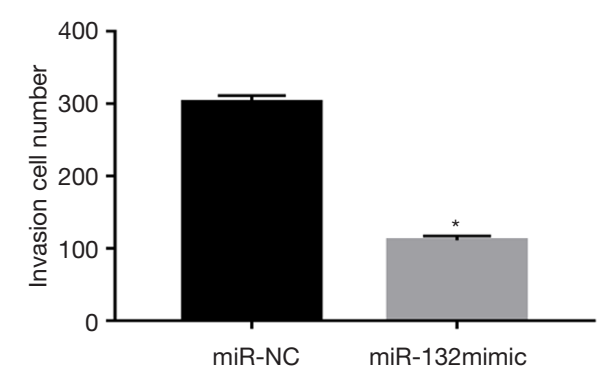

Figure 3 miR-132 inhibits the migration and invasion ability of ovarian cancer cells. (A) miR-132 inhibited migration in HO-8910 cells, scratch assay: magnification, $\times 40$. * $\mathrm{P}<0.05$ compared with miR-NC. (B) The results of Transwell chamber invasion assay showed that miR132 inhibited the invasion ability of HO-8910 cells. Transwell chamber invasion assay: magnification, $\times 100$. *, $\mathrm{P}<0.05$ in comparison with miR-NC. miR, microRNA; NC, negative control; miR-132, microRNA-132.

overexpression in HO-8910 cells.

Further, we also studied CRKII expression in 40 pairs of ovarian cancer tissues and normal tissues with qRT-PCR. As expected, the ovarian cancer tissues had a higher expression of CRKII than the normal tissues (Figure 4E,F), which was negatively correlated with miR-132 expression. These findings indicate that CRKII is a direct target of miR-132 in ovarian cancer. 
A

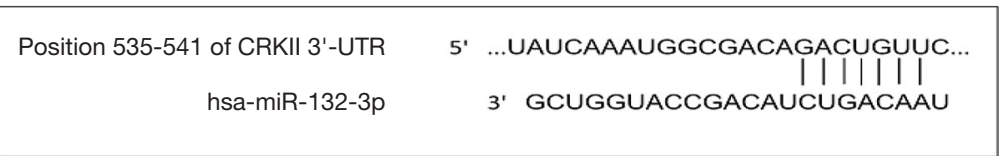

B

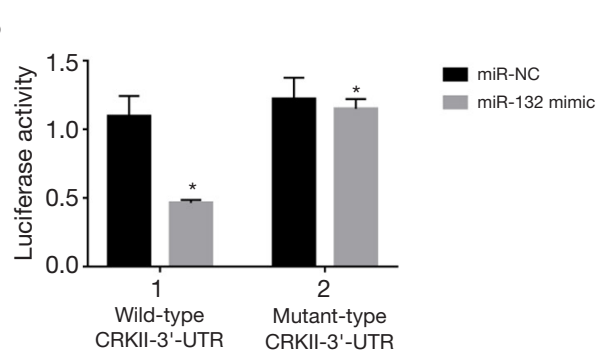

C

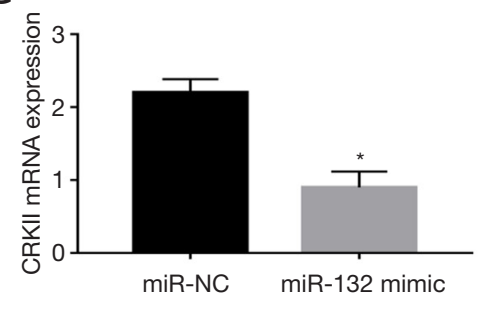

D



E

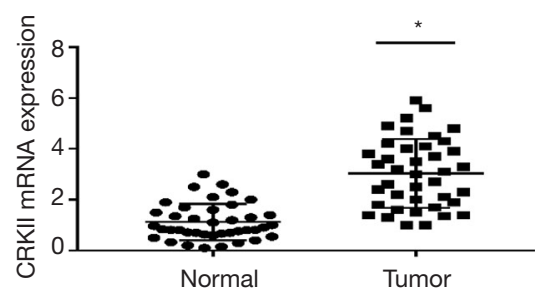

$\mathrm{F}$

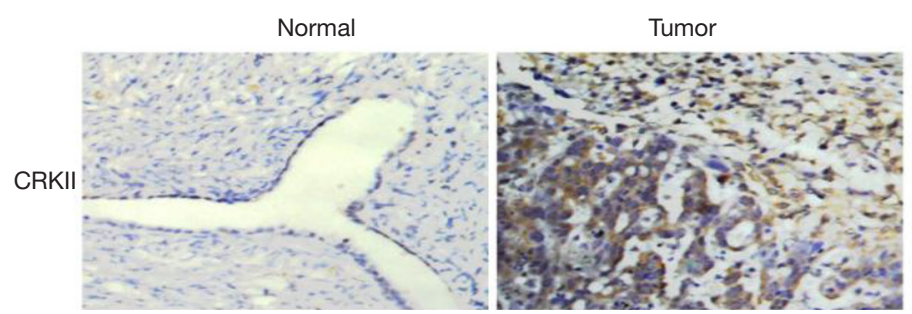

Figure 4 CRKII is a direct target of miR-132 in ovarian cancer. (A) The predicted binding sites for miR-132 in the 3'-UTR of CRKII (positions 535-541) are shown. (B) After 48 hours, luciferase activities in HO-8910 cells co-transfected with wide-type CRKII 3'UTR luciferase plasmid or mutant-type CRKII 3'-UTR luciferase plasmid and miR-132 mimic or miR-NC. *, $\mathrm{P}<0.05$ in comparison to miR-NC. (C) CRKII mRNA expression in miR-132 mimic or miR-NC-transfected HO-8910 cells was determined by qRT-PCR. *, $\mathrm{P}<0.05$ compared with miR-NC. (D) The CRKII protein level in miR-132 mimic or miR-NC-transfected HO-8910 cells was determined by Western blot. (E) The expression of CRKII mRNA in 40 ovarian cancer tissues and corresponding normal tissues was detected by qRT-PCR. *, P $<0.05$ compared to normal samples. (F) Detection of CRKII expression in ovarian cancer tissues and cells by immunohistochemistry (400 times magnification). CRKII, CT10 Oncogenic Gene Homologue II; miR-132, microRNA-132; qRT-PCR, quantitative reverse transcription polymerase chain reaction.

\section{Loss of CRKII inhibits the proliferation, colony-forming, migration, and invasion abilities of ovarian cancer cells}

To explore the role of CRKII in ovarian cancer, siCRKII or si-NC was transfected into HO-8910 cells. The mRNA and protein expressions of si-CRKII and si-NC in HO-8910 cells were detected by qRT-PCR and Western blot. As shown in Figure $5 A, B$, compared with the cells transfected with $\mathrm{Si}-\mathrm{NC}$, the expression of CRKII in the Si-CRKII-transfected cells was decreased. We also discovered that the down-regulation of CRKII significantly inhibited cell proliferation, colony formation, migration, and invasion in HO-8910 cells (Figure 5C,D,E,F), mimicking the effect of mir-132 overexpression. 

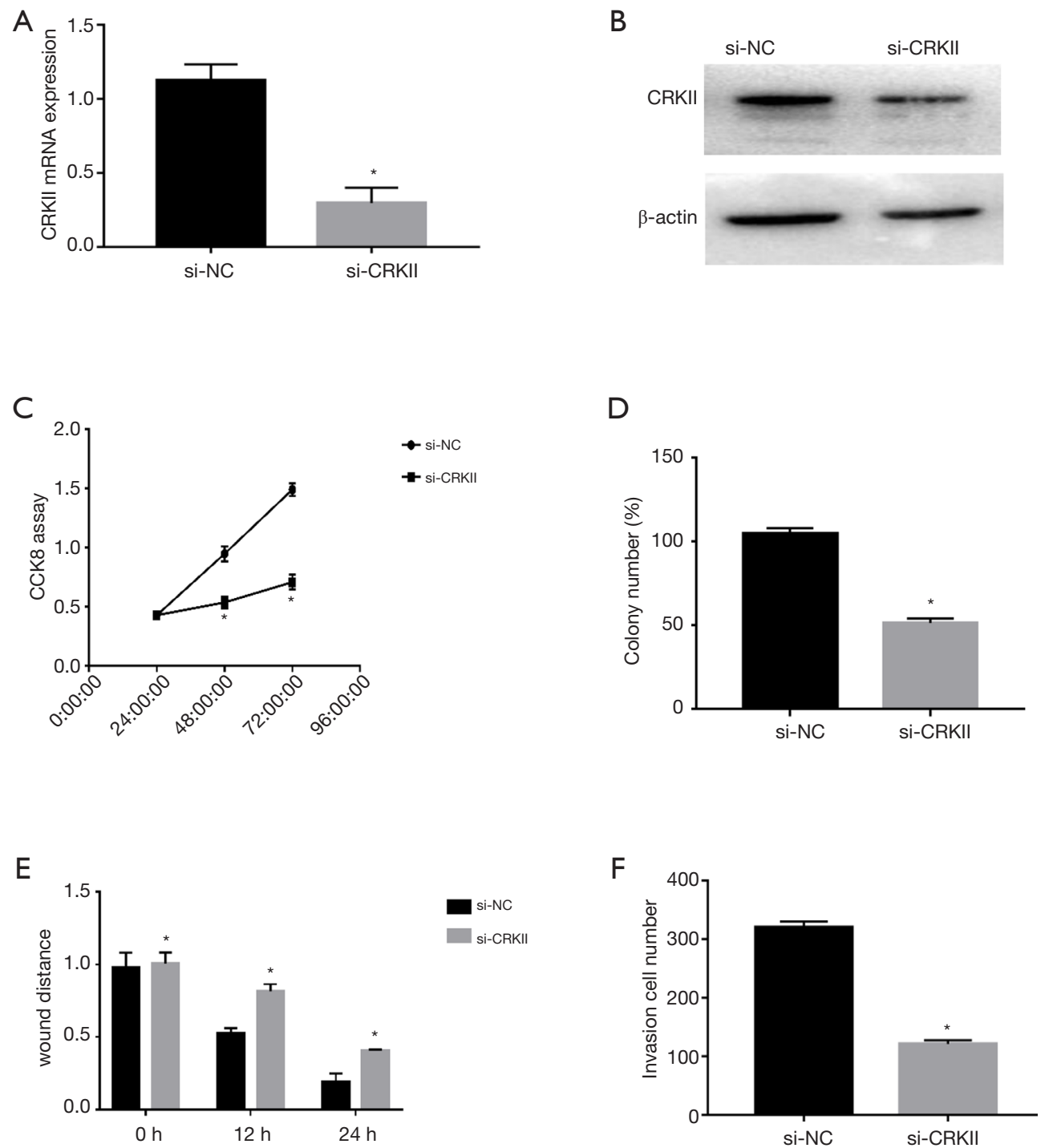

Figure 5 The deletion of CRKII inhibited the proliferation, colony formation, migration, and invasion of ovarian cancer cells. mRNA (A) and protein (B) levels of CRKII in HO-8910 cells transfected with si-CRKII or si-NC were determined by qRT-PCR and Western Blot. In HO-8910 cells transfected with si-CRKII or si-NC, cell proliferation (C), colony formation (D), migration (E), and invasion (F) were detected. *, P<0.05 compared with si-NC. CRKII, CT10 Oncogenic Gene Homologue II; si-CRKII, siRNA against CRKII; si-NC, siRNA against negative control; qRT-PCR, quantitative reverse transcription polymerase chain reaction.

miR-132 inbibits cell proliferation, colony formation, migration, and invasion in ovarian cancer cells by targeting CRKII

To better understand the activity of miR-132 in targeting CRKII in ovarian cancer cells, we transfected HO-8910 cells with miR-132 mimic or miR-NC along with vectorcontrolled (pcDNA3) or overexpressed CRKII plasmid (pcDNA3-CRKII). The results of qRT-PCR and Western blot showed that the co-transfection of pcDNA 3-CRKII and miR-132 could restore the expression level of CRKII (Figure 6A,B) compared with the control vectors with miR132. Moreover, we discovered that CRKII overexpression plasmid could offset the adverse effects of miR-132 on ovarian cancer cell hyperplasia colony formation, migration, and invasion (Figure 6C,D,E,F). Overall, these findings indicated that miR-132 inhibits cell proliferation, migration, and invasion in ovarian cancer by regulating CRKII. 


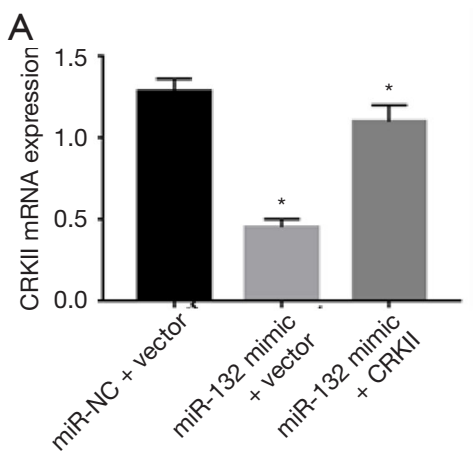

B


Figure 6 miR-132 inhibits cell proliferation, colony formation, migration, and invasion by targeting the expression of CRKII. Detection of CRKII mRNA (A) and protein (B) levels in HO-8910 cells transfected with miR-132 mimics or NC, and detection of vector control (pcDNA3) or overexpressed CRKII plasmids (pcDNA3-CRKII) levels. HO-8910 cells transfected with miR-132 mimics or NC were detected for cell proliferation (C), colony formation (D), migration (E), and invasion (F), as well as vector control (pcDNA3) or overexpressed CRKII plasmids (pcDNA3-CRKII). *, P<0.05 compared with cells transfected with miR-132 plus the control vector. CRKII, CT10 Oncogenic Gene Homologue II; miR-132, microRNA-132; NC, negative control.

\section{Discussion}

Many miRNAs have been shown to be involved in the occurrence, development, and metastasis of ovarian cancer through targeting many key protein-coding genes (11). For instance, the expression of TUSC2 was negatively correlated with the level of miR-663 in ovarian carcinoma tissue, while TUSC2 overexpression inhibited the migration and invasion abilities of SKOV3 after promotion by miR663 (12). By inactivating of $\mathrm{Wnt} / \beta$-catenin signaling pathway, miR-16 inhibits the migration and invasion of ovarian cancer cells in vitro (13). When down-regulated, miR-183 has been shown to significantly inhibit the proliferation, migration, and invasion of ovarian cancer 
cells and to promote apoptosis, regulate Smad4 through the TGF- $\beta /$ Smad 4 signaling pathway, and play a tumorpromoting role in ovarian cancer (14). miR-34 is downregulated in human ovarian cancer cells. The proliferation of ovarian cancer cells is inhibited by mir-34 overexpression, which induces apoptosis and autophagy by targeting Notch 1 (15). In our study, we found miR-132 expression to be significantly decreased in ovarian cancer tissues and cell lines, and proved that miR-132 had an inhibitive effect on the growth, migration, and invasion of ovarian cancer cells. Our findings suggest that miR-132 plays a role in the development and progression of ovarian cancer.

miRNA plays the dual role of oncogene and tumor suppressor, and its expression level is closely tied to tumor occurrence and development. Currently, miR-132 is one of the hotspots in miRNA-related research. miR-132 is not only down-regulated in lung cancer, gastric cancer, and ovarian cancer, but it is also closely associated with the occurrence and development of malignant tumors. Moreover, it is expected to be a new target for the inhibition of malignant tumors. Stuckrath et al. showed in breast cancer patients that abnormal regulation of miR-132 is related to poor prognosis and lymph node metastasis (16). Khan et al. also demonstrated that after treatment with omexifene and gemcitabine, the expression of miR132 in xenograft tumors was significantly up-regulated and the evolution of stromal cells into tumor tissues was inhibited (17). Meanwhile, Wang et al. found that miR132 was significantly under-expressed in osteosarcoma and could inhibit tumor proliferation by targeting the regulation of Cyclin E1 (18). All of these studies confirmed the importance of miR-132 in the regulation of tumor progression through its targeting of related molecules. In ovarian cancer, microarray analysis by Chung et al. found that miR-132 expression in serous ovarian cancer patients was significantly lower compared to that in healthy individuals and that it can be considered as a potential novel biomarker of serous ovarian cancer (19). Nevertheless, the detailed functions and molecular mechanisms of miR-132 in ovarian cancer have yet to be fully remain illuminated. In this study, we showed that the level of miR-132 was significantly reduced in ovarian cancer tissues and cell lines, and that miR-132 overexpression in ovarian cancer cells reduced cell proliferation, colony formation, migration, and invasion in vitro. These findings indicate that miR-132 may serve as a tumor suppressor in the progression of ovarian cancer.

miRNAs are widely understood to exert their biological functions by regulating target genes (20). In recent years, an increasing number of studies have shown that CRKII expression is closely linked with the occurrence and development of tumors. Multiple extracellular factors stimulate the corresponding receptors, receptor tyrosine phosphorylation, or focal adhesion components, such as p130Cas and pile protein phosphorylation. Then, through the $\mathrm{SH} 2$ domain structure prompt CRKII nearly to raise membrane area, position change CRKII can induce Rap1, Rasm and Rac activation, and the regulation of tumor cell adhesion, proliferation, and migration via its $\mathrm{SH} 3$ domain structure and downstream proteins such as proline-rich C3G, SOS, and DOCK180 (21). Lamorte et al. showed that CRKII with localization changes can also promote epithelial mesenchymal transformation in renal epithelial cell tumors and is necessary for hepatocyte growth factor-mediated cell proliferation and migration (22). Cheung et al.'s study on non-small cell lung cancer CRKII was shown to competitively activate Src through direct binding with CSK and induce the phosphorylation of downstream transcription factor STAT5 to activate CyclinD1 and enhance cell proliferation (23). Wang et al. confirmed that CRKII expression was significantly up-regulated in SKOV3 (24). Furthermore, its overexpression promoted cell proliferation, while knockdown of CRKII expression with siRNA technology achieved the opposite results.

In summary, the results of this study showed that the expression level of miR-132 was decreased in ovarian cancer tissues and cell lines. The overexpression of miR-132 significantly inhibited cell proliferation, colony formation, migration, and invasion through the inhibition of CRKII. The findings of our study suggest that miR-132 acts like a tumor suppressor in ovarian cancer via targeting CRKII, and that miR-132 could potentially be an important target in treating ovarian cancer.

\section{Acknowledgments}

Funding: None.

\section{Footnote}

Reporting Checklist: The authors have completed the MDAR reporting checklist. Available at http://dx.doi.org/10.21037/ tcr-20-2435

Data Sharing Statement: Available at http://dx.doi. org/10.21037/tcr-20-2435 
Conflicts of Interest: All authors have completed the ICMJE uniform disclosure form (available at http://dx.doi. org/10.21037/tcr-20-2435). The authors have no conflicts of interest to declare.

Ethical Statement: The authors are accountable for all aspects of the work in ensuring that questions related to the accuracy or integrity of any part of the work are appropriately investigated and resolved. This study was conducted in adherence with the guidelines of the Declaration of Helsinki (as revised in 2013) and received approval from the Ethics Committee of Nantong University (2019-L060). Written informed consent was obtained from all patients.

Open Access Statement: This is an Open Access article distributed in accordance with the Creative Commons Attribution-NonCommercial-NoDerivs 4.0 International License (CC BY-NC-ND 4.0), which permits the noncommercial replication and distribution of the article with the strict proviso that no changes or edits are made and the original work is properly cited (including links to both the formal publication through the relevant DOI and the license). See: https://creativecommons.org/licenses/by-nc-nd/4.0/.

\section{References}

1. Torre LA, Trabert B, DeSantis CE, et al. Ovarian cancer statistics, 2018. CA Cancer J Clin 2018;68:284-96.

2. Xue C, Zhu D, Chen L, et al. Expression and prognostic value of PD-L1 and PD-L2 in ovarian cancer. Transl Cancer Res 2019;8:111-9.

3. Gorodnova TV, Sokolenko AP, Kuligina E, et al. Principles of clinical management of ovarian cancer. Chin Clin Oncol 2018;7:56.

4. Nudelman AS, DiRocco DP, Lambert TJ, et al. Neuronal activity rapidly induces transcription of the CREBregulated microRNA-132, in vivo. Hippocampus 2010;20:492-8.

5. Srivastava SK, Ahmad A, Zubair H, et al. MicroRNAs in gynecological cancers: Small molecules with big implications. Cancer Lett 2017;407:123-38.

6. Cambronne XA, Shen R, Auer PL, et al. Capturing microRNA targets using an RNA-induced silencing complex (RISC)-trap approach. Proc Natl Acad Sci U S A 2012;109:20473-8.

7. Lin L, Wang Z, Jin H, et al. MiR-212/132 is epigenetically downregulated by SOX4/EZH2-H3K27me3 feedback loop in ovarian cancer cells. Tumour Biol 2016. [Epub ahead of print].

8. Kumar S, Lu B, Davra V, et al. Crk Tyrosine Phosphorylation Regulates PDGF-BB-inducible Src Activation and Breast Tumorigenicity and Metastasis. Mol Cancer Res 2018;16:173-83.

9. Bell ES, Park M. Models of crk adaptor proteins in cancer. Genes Cancer 2012;3:341-52.

10. Kobashigawa $Y$, Sakai M, Naito M, et al. Structural basis for the transforming activity of human cancer-related signaling adaptor protein CRK. Nat Struct Mol Biol 2007;14:503-10.

11. Chen SN, Chang R, Lin LT, et al. MicroRNA in Ovarian Cancer: Biology, Pathogenesis, and Therapeutic Opportunities. Int J Environ Res Public Health 2019;16:1510.

12. Xie HH, Huan WT, Han JQ, et al. MicroRNA-663 facilitates the growth, migration and invasion of ovarian cancer cell by inhibiting TUSC2. Biol Res 2019;52:18.

13. Li N, Yang L, Sun Y, et al. MicroRNA-16 inhibits migration and invasion via regulation of the $\mathrm{Wnt} /$ betacatenin signaling pathway in ovarian cancer. Oncol Lett 2019;17:2631-8.

14. Zhou J, Zhang C, Zhou B, et al. miR-183 modulated cell proliferation and apoptosis in ovarian cancer through the TGF-beta/Smad4 signaling pathway. Int J Mol Med 2019;43:1734-46.

15. Jia $\mathrm{Y}$, Lin R, Jin H, et al. MicroRNA-34 suppresses proliferation of human ovarian cancer cells by triggering autophagy and apoptosis and inhibits cell invasion by targeting Notch 1. Biochimie 2019;160:193-9.

16. Stuckrath I, Rack B, Janni W, et al. Aberrant plasma levels of circulating miR-16, miR-107, miR-130a and miR-146a are associated with lymph node metastasis and receptor status of breast cancer patients. Oncotarget 2015;6:13387-401.

17. Khan S, Ebeling MC, Chauhan N, et al. Ormeloxifene suppresses desmoplasia and enhances sensitivity of gemcitabine in pancreatic cancer. Cancer Res 2015;75:2292-304.

18. Wang J, Xu G, Shen F, et al. miR-132 targeting cyclin E1 suppresses cell proliferation in osteosarcoma cells. Tumour Biol 2014;35:4859-65.

19. Chung YW, Bae HS, Song JY, et al. Detection of microRNA as novel biomarkers of epithelial ovarian cancer from the serum of ovarian cancer patients. Int J Gynecol Cancer 2013;23:673-9.

20. Ohtsuka M, Ling H, Doki Y, et al. MicroRNA Processing 
and Human Cancer. J Clin Med 2015;4:1651-67.

21. Liu D. The adaptor protein Crk in immune response. Immunol Cell Biol 2014;92:80-9.

22. Lamorte L, Rodrigues $S$, Naujokas $M$, et al. Crk synergizes with epidermal growth factor for epithelial invasion and morphogenesis and is required for the met morphogenic program. J Biol Chem 2002;277:37904-11.

23. Cheung HW, Du J, Boehm JS, et al. Amplification of CRKL induces transformation and epidermal growth

Cite this article as: Jiang H, Dai M, Wu Y, Dong Y, Qi L, Xi Q, Liang G. microRNA-132 inhibits the proliferation, migration, and invasion of ovarian cancer cells by regulating CT10 oncogenic gene homologue II-related signaling pathways. Transl Cancer Res 2020;9(7):4433-4443. doi: 10.21037/tcr-202435 factor receptor inhibitor resistance in human non-small cell lung cancers. Cancer Discov 2011;1:608-25.

24. Wang H, Linghu H, Wang J, et al. The role of Crk/ Dock180/Rac1 pathway in the malignant behavior of human ovarian cancer cell SKOV3. Tumour Biol 2010;31:59-67.

(English Language Editor: J. Reynolds) 